Témoigner Témoigner. Entre histoire et mémoire

Getuigen Revue pluridisciplinaire de la Fondation Auschwitz

$124 \mid 2017$

La musique dans les camps

\title{
Requiem pour un massacre d'Elem Klimov. Le film de guerre russe « oublié »
}

\section{Brecht Capiau}

Traducteur : Emilie Syssau

\section{OpenEdition \\ Journals}

\section{Édition électronique}

URL : https://journals.openedition.org/temoigner/5663

DOI : $10.4000 /$ temoigner.5663

ISSN : 2506-6390

\section{Éditeur :}

Éditions du Centre d'études et de documentation Mémoire d'Auschwitz, Éditions Kimé

Édition imprimée

Date de publication : 2 avril 2017

Pagination : 13-16

ISBN : 978-2-930953-00-7

ISSN : 2031-4183

\section{Référence électronique}

Brecht Capiau, «Requiem pour un massacre d'Elem Klimov. Le film de guerre russe « oublié » ,

Témoigner. Entre histoire et mémoire [En ligne], 124 | 2017, mis en ligne le 30 novembre 2021, consulté le 01 décembre 2021. URL : http://journals.openedition.org/temoigner/5663; DOI : https://doi.org/ 10.4000/temoigner.5663 


\section{REQUIEM POUR UN MASSACRE D'ELEM KLIMOV LE FILM DE GUERRE RUSSE “OUBLIÉ »}

DVD Quand II ouvrit le quatrième sceau, jentendis la voix du quatrième être vivant qui disait : «Viens et vois! » Et voici que parut un cheval d'une couleur pâle. Celui qui le montait se nommait la mort, et le séjour des morts l'accompagnait. Le pouvoir leur fut donné sur le quart de la terre, pour faire périr les hommes par l'épée, par la famine, par la mortalité, et par les bêtes sauvages de la terre. (Apocalypse de Jean ou Livre de la révélation)

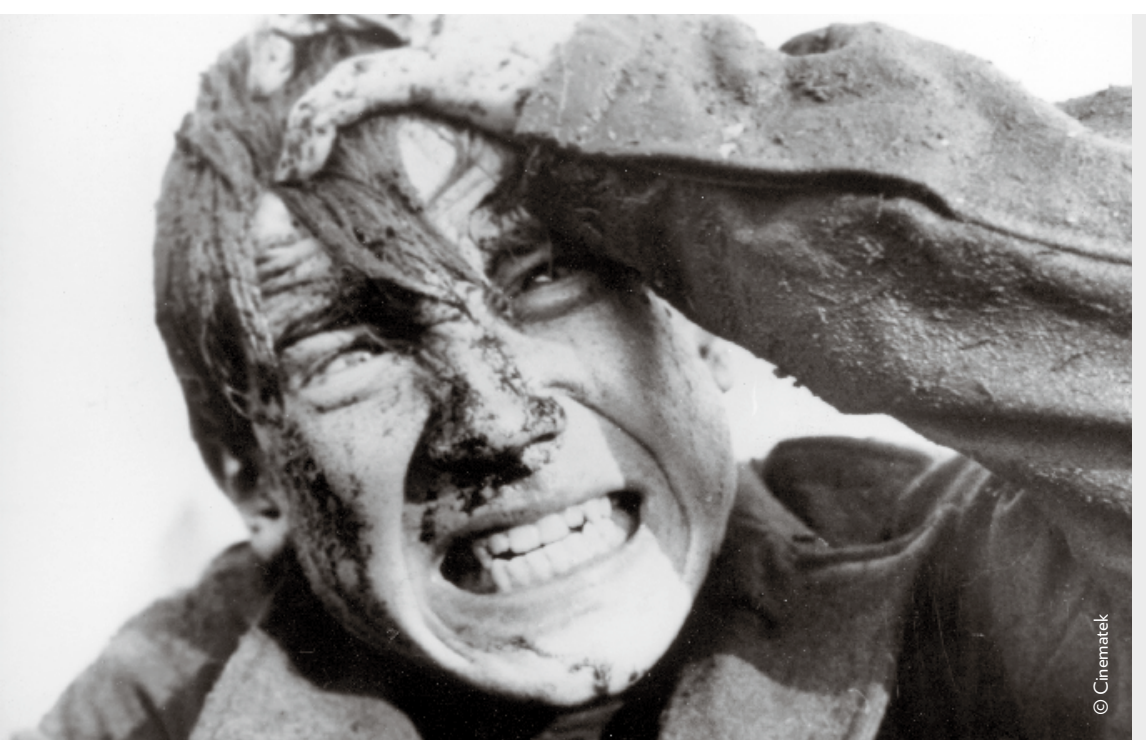

Projection le dimanche 30 mai dans le cadre du Ciné-club de la Mémoire d'Auschwitz ASBL.

Le thème de l'année 2017 : Une enfance perdue.

$$
\begin{gathered}
\text { Plus d'infos : } \\
\text { Passeurs d'images } \\
\text { www.cineclub.brussels } \\
\text { Cinéma AVENTURE } \\
\text { Galerie du Centre } \\
\text { Rue des Fripiers } 15 \\
1000 \text { Bruxelles }
\end{gathered}
$$

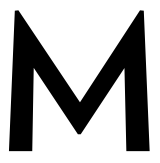

oscou, 9 mai 2015. La Russie célèbre par un impressionnant défilé militaire le $70^{\mathrm{e}}$ anniversaire de sa victoire surl'Allemagne nazie. Plus de 16000 soldats marchent solennellement sur la Place Rouge. Malgré la présence d'une vingtaine de chefs d'État et de nombreux badauds qui se pressent contre les barrières de sécurité, Poutine se sent relativement «seul». De nombreux pays occidentaux lui ont en effet posé un lapin. Cet acte politique peut être interprété de plusieurs manières. D’abord, l'Occident manifeste avec force sa désapprobation de l'annexion de la Crimée et somme Poutine de réfréner ses ambitions. Mais l'absence des dirigeants européens peut aussi donner l'impression qu'ils se désintéressent des souffrances du peuple soviétique durant la Seconde Guerre mondiale. Si la première motivation est à encourager, la seconde est regrettable.

Personne ne contestera que l'Union soviétique a fait d'immenses sacrifices pendant la Seconde Guerre mondiale. On peut certes s'interroger sur les relations que la Russie et l'Europe occidentale entretiennent avec ce passé, et se demander dans quelle mesure on peut parler de récupération politique. Aux yeux des $\bullet \bullet$ 
•. Occidentaux, tous les produits culturels (notamment films et livres) de l'URSS sur cette époque sont un forme de «propagande soviétique». L'URSS, de son cote, s'enployait avec acharnent à entretenir mythe de la "Grande Guere patriotique». Le film Requiem port [iteralement Viens et vois, titre frança Rlequiem pour un massace] réalisé en 1985 par Elem limovestatypiquè cet égard:íne sinscrit pas dan a prande Guerre patriotiquen. Il racopte la «Grande Guerre patriotique». Il raconte en outre ve historie du point dev victimes d'une guerre.

LE PREMIER SCEAU : LA GENÈSE

Requiem pour un massacre sort à l’occasion du quarantième anniversaire de la fin de la Seconde Guerre mondiale. Ce film est le fruit de l'engagement profesinnel et émotionnel de son réalisateur Elem Klimor et de son scénariste Ales Adamovitch. Tous deux on connul horreur nazie qui y est présentée. Né en 1933 de ville par la Wehrmacht allemande à lété du siège dectobre de cette même année, il fuitla ville 1942. En avec sa ère et son frìre German. Ils franchiss vec sa mère et son frère German. Ils franchissent la

Volga sur un radeau improvisé et cherchent leur salut dans les monts Oural. Klimov est marqué à vie par ce ill a vécu durant sa fuite. Dans les entretiens qu'il a a propos de Requiem pour un massacre, son opus le plus célèbre, il apparaît rapidement que ce film tient ont il a été témoin.

Klimov a également été poussé à faire ce film en aison de la situation politique dans l'Union soviétique

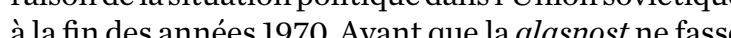
son apparition au milieu des années 1980, las froide touche à son paroxysme. Klimov voit s’élever le fantôme d'une Troisième Guerre mondite le fantive à faire un film signicatif En 1975 par le motive à faire un film significatif. En 1975 paraît d'abord son Raspoutine, l'agonie, extraordinairement baroque et somptueux. Ce film, qui esquisse un portrai psychologicue de Raspoutine et de la famille de Nicoen raisto du en raison du regard trop nuancé qu il offre sur Nicolas limov àcerd to ónest mour en scène. Désappointé, demer réalisatrice dufilm L'Asces Larissa Shepiko, célebre cident de voiture.

Klimov parvient finalement à se reprendre. Son nouveau film doit surpasser Raspoutine, l'agonie sur le plan tant artistique que psychologique et assurer son come-back. La rencontre avec l'ecrivain Ales Adamovitch accélère les choses. Les deux hommes se rejoignent par la similarite de leur expérience de la guerre. Adamovitch conserve du temps passé
chez les partisans en Biéchez les partisans en Biélorussie un bagage émotionnel qui donne une au projet du flenture au projet du film. Une première version du scénario est intitulée Tuez Hitler invitation à ne jamais à la peur de l'autre. Goskino, le comité d'État pour le cinéma, ne veut cependant pas cautionner le titre et e réalisme du film. Lidée semble mourir d une douce mort. Puis le projet bénéficie dun nouvel élan quan German, le frère dElem Klinov, propose le titre Idi Il faudra

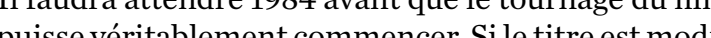
pí, Klimov et Adamovitch ne font aucunecons. au scénario ou au style réaliste.

LE DEUXIĖME SCEAU : LINNNOCENCE DE L'ENFANCE CONFRONTÉE À LA PERVERSION DES ADULTES

Fixer harmonieusement sur la pellicule l'expérience d'un enfant pendant la guerre est une entreprise délicate pour Elem Klimov. Il était pour lui primordial que le jeune protagoniste de treize ans, Alexeï Kravchenko, ne souffre d'aucun traumatisme en cours de tournage et sache resituer ses prestations dans le cadre du film. Dans les bonus de l'édition DVD de Ruscico, le réalisateur raconte qu'il a notamment fait appel à des hypnotiseurs pour déceler et tester l'inconscient du garçon, et le protéger des scènes qui lattendaient. garçon, et le protéger des scènes qui lattendaient.

Le film s'ouvre sur Fliora en train de jouer sur la plage avec un copain. La scène prend rapidement un tour sinistre quand on découvre qu'ils ne construisent pas un château de sable, mais déterrent des arme enfouies. Un paysan du village les prévient qu'ils feraient mieux de laisser reposer le passé - premièr opposition entre linnocence de lenfance et la perLs enfants Les enfants sont jeunes et imprudents, le paysan est vieux et a vécu. Avec larme pour symbole de prestige, le garçon veut rejoindre les «courageux» partisans. ta scène ou thora pose en manteau nér, sac à dos et de son premier jour de classe.

C'estle début d'une odyssée cauchemardesque inviant à explorer les limites de la résistance mentale tant à explorer les limites de la résistance mentale et
physique. Âla fin du film, le garçon est un enfant vieilli, physique. Ala láin du film, le garçon est un enfant vieilli, Lorsquon lui donne la chance de se venger symboliLorsqu'on lui donne la chance de se venger symboliquement tu grand instigateur de toute cette violence, un port it du dictateur dans lespir. de rait feu sur ituation. Il s'ensuit un effet de rembobinage de l'histoire : on remonte dans le temps, et on voit des bombe retour 'Allemagne, et Hitler se tranformer d'home enfant. L' '’u d'Hitler bébé incite Fliora à me en un terme à la upunition»: il comprend à cet instrent 政 de leur futur moi. Telle est la conséquence ultimase the du film.

LE TROISIĖME SCEAU : LA CINÉMATOGRAPHIE DU FILM

Le réalisateur Klimovutilise en 1985 une technique cinématographique nouvelle pour le cinéma soviétique qui fait son effet sur grand écran. Il utilise abondammentla steady cam, une caméra fixée sur le caméraman, e qui donne l'impression que la caméra glisse sur le champs et les villes sablonneuses de Biélorussie. Nous parlons aussi dans ce cas d'une caméra subjective, le pectateur ayant le sentiment de courir avec les personnages, d'endurer ce qu'ils endurent et de pouvoir eter un regard intime sur des événements difficiles o de caméra.

Requiem pour un massacre est aussi connu pour jouer intelligemment avec des «images intérieures Le film compote donc une prise de vue qui donne l'impression que Fliora a été exécuté. Mais ce n'est Mupression que Fiora a été exécuté. Mais ce n'est à voir que les soldats mlattent en scène l'exécution à l'intention d'un photographe. Les personexestion a bour

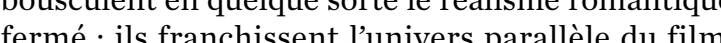
ermé : ils franchissent lunivers parallèle du film, les yeux du spectateur (voir Cousins, 2005). Avec cette

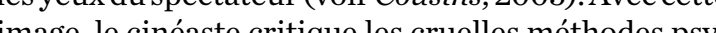
mage, le cinéaste critque les cruelles méthodes psyvictimes.

LE QUATRIĖME SCEAU : RÉCEPTION CRITIQUE ET PLACE DANS LE CINÉMA SOVIÉTIQUE

Après sa sortie, Requiem pour un massacre doit essuyer les vives critiques de quelques journalistes, qui ylisent un message prorusse caché. Ils pensent que 年 


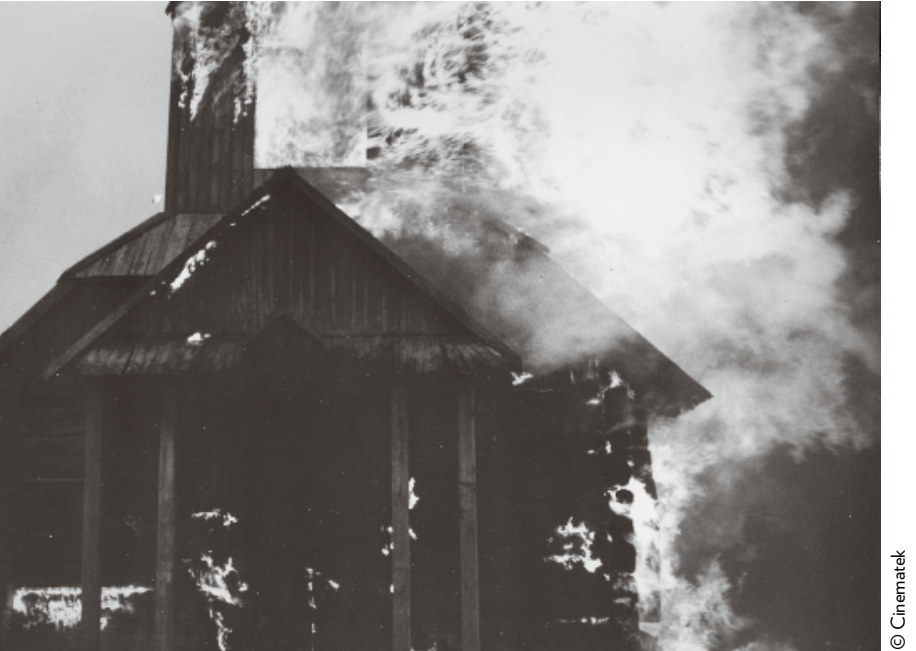

• En Belgique, le journaliste de cinéma Piet Goethals est partisan de cette thèse, qu'il formule ainsi :

Katyn constitue un problème historique en soi, puisque 4500 officiers polonais y ont été assassinés par des staliniens. Les Russes l'ont toujours nié et en ont attribuéla culpabilité aux nazis. [...] Klimovjoue sur cette ambiguïté historique et prend le parti des Russes sous le couvert d'un film antifasciste (Goethals, 1988, p. 32-33).

Dans les bois de Katyn, près de la ville de Smolensk, le NKVD (police secrète de l'URSS) a effectivement abattu en avril et en mai 1940 des milliers de membres de l'intelligentsia militaire polonaise. Les nazis ont découvert les charniers en 1941 lors de leur conquête de l'Union soviétique. Les autorités soviétiques devaient contester ce crime jusqu'en 1990, et Goethals estimait que Klimov cautionnait avec son film le discours de Moscou de l'époque.

Il s'agit cependant ici d'une méprise, car Klimov a basé son film sur le livre The Book of Khatyn d'Adamovitch. Khatyn (avec un h) est un village de Biélorussie qui a été entièrement dévasté en 1943 par le $118^{\mathrm{e}}$ bataillon de laSchutzmannschaft formée par la Hilfspolizei ukrainienne. Avec seulement une lettre de différence, l'erreur est vite commise - et cela souligne d'autant plus le fait que des films comme Requiem pour un massacre doivent être regardés avec une plus grande attention pour pouvoir comprendre pleinement leur valeur historique.
Dans les années 1980, le cinéma soviétique ne répondait plus depuis longtemps aux règles du réalisme socialiste prescrites par Staline. Requiem pour un massacre est le point final d'une évolution commencée peu après la mainmise de Staline sur le pouvoir. Des chercheurs comme Denise Youngblood soulignent que Quand passent les cigognes (Kalatozov, 1957), L'Enfance d'Ivan (Tarkovski, 1962), L'Ascension (Shepitko, 1977) et Requiem pour un massacre (Klimov, 1985) ont réussi à créer une mémoire authentique du conflit :

En dépouillant la guerre de l'hypocrisie et de la grandiloquence de l'histoire officielle, en confrontant ses nombreux paradoxes à l'infaillible honnêteté, ces réalisateurs ont réussi là où les historiens avaient échoué (et ils ne pouvaient d'ailleurs réussir, étant donné les restrictions de la profession d'historien dans l'Union soviétique) (Youngblood, 2001, p. 855, traduction E.S.).

Requiem pour un massacre appartient à la liste très restreinte des œuvres cinématographiques qui ne servent aucun programme politique et que nous pouvons même cataloguer comme films antiguerres. I

Brecht Capiau

Traduit du néerlandais par Emilie Syssau

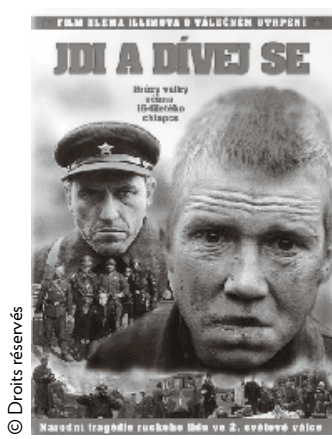

\section{Plus d'infos}

Elem Klimov, Requiem pour un massacre [DVD], Ruscico, 2000, $145 \mathrm{~min}$.

\section{Bibliographie}

Mark Cousins, Close-up: een wereldgeschiedenis van de film, Amsterdam, Van Holkema \& Warendorf, 2005.

Piet Goethals, « Idi i smotri, Die Leiden des jungen Florya », Filmmagie 371, 1988, p. 32-33.

Denise Youngblood, «A War Remembered: Soviet Films of the Great Patriotic War », The American Historical Review 106 (3), 2001, p. 839-856. 